\title{
Strategies of diagnosis and treatment for patients with diabetic foot ulcer during coronavirus disease (COVID-19) pandemic
}

Jinying Zhang

The Second Affiliated Hospital of Fujian Medical University

Jiayu Lin ( 429557370@qq.com )

The Second Affiliated Hospital of Fujian Medical University

\section{Research Article}

Keywords: 2019-nCoV, Diabetes, Diabetic foot ulcer, Vacuum sealing drainage

Posted Date: May 17th, 2022

DOI: https://doi.org/10.21203/rs.3.rs-1338366/v2

License: (c) (1) This work is licensed under a Creative Commons Attribution 4.0 International License.

Read Full License 


\section{Abstract \\ Background}

The coronavirus disease 2019(COVID-19) pandemic is breaking out and rapidly spread across the country, which is also a severe ordeal for medical workers, and the traditional treatment strategy of diabetic foot ulcer (DFU) is also facing challenges.

\section{Methods}

We herein report four patients with diabetic foot ulcers during the COVID-19 pandemic who achieved individual diagnosis and treatment plan, made patients get timely and reasonable treatment on the premise of prevention and control. After treatment, the wound healed, and the foot function recovered.

\section{Results}

After treatment, the wound healed, and the foot function recovered.

\section{Conclusions}

According to the guidelines of relevant domestic experts, this paper recommends reasonable recommendations for the diagnosis and treatment of diabetic foot ulcers, optimizing the diagnosis and treatment process, and reducing cross-infection in the hospital.

\section{Introduction}

Diabetic foot ulcer (DFU) is a chronic severe complication in the late stage of diabetes mellitus, which is the common cause of amputation or disability of lower limbs in diabetic patients[1]. With the incidence rate of diabetes increasing, the incidence of foot ulcers also increases, which is about $5 \%$ to $8 \%[2]$. The amputation rate of diabetic foot patients is as high as $20 \%$, and DFU amputations account for $27.3 \%$ of all amputations, accounting for $56.5 \%$ of nontraumatic amputation[3]. The fear of amputation in diabetic foot patients is often far more significant than death, foot infection, and end-stage renal disease[4].

Diabetic foot infection (DFIs) usually starts from a small wound that is not easy to find by patients and then develops into a more complex wound due to peripheral neuropathy[5]. Concurrent local microcirculation disturbance, arteriosclerotic stenosis of the lower extremities, in addition to decreased immune ability, and prolonged wound infection are risk factors associated with wound nonunion, forming a chronic or refractory ulcer, and even amputation, which bring great pain to the patients. Vacuum sealing drainage(VSD) can effectively promote the safe and rapid repair of large-area skin defects, and provide a good prognosis [6]. 
In December 2019, the epidemic of COVID-19 (COVID-19) broke out in Wuhan, Hubei Province, and rapidly spread across the country. Mounting cases and deaths brought about significant public health and governance challenges. A recent retrospective study of more than 40,000 diagnosed COVID-19 patients showed that patients with underlying diseases such as hypertension, diabetes, or cardiovascular disease were more likely to be infected by $2019-\mathrm{nCoV}$ and had a high mortality rate. Of the more than 20, 000 COVID-19 patients with underlying disease records, $12.8 \%$ were complicated with hypertension, and $5.3 \%$ with diabetes. Among the dead COVID-19 patients, $22.7 \%$ were complicated with cardiovascular disease and $19.7 \%$ with diabetes[7]. Thus it can be seen that people with diabetes are not only a high-risk group of COVID-19 but also a high-risk group of death. The COVID-19 pandemic has presented many challenges in the management of people with diabetes. During the COVID-19 pandemic, the medical resources of diagnosis and treatment of diabetic foot reduced relatively. In contrast, most diabetic foot patients need long-term outpatient follow-up, and some patients need operation within deadline or emergency operation. According to the new situation, medical staff needs to take new diagnoses and treatment procedures and measures for diabetic foot patients.

Immediately after all DFU patients arrive at the outpatient clinic, the triage nurse inquires about the epidemiological history and take the body temperature, and then conduct the first COVID-19 screening. The receiving physician still needs to ask the epidemiology history in detail, including whether there are clinical symptoms such as sore throat, fever, cough, expectoration, diarrhea, and so on, and screen again for the possibility of COVID-19. The nurses of the diagnosis area should accompany DF patients to the fever clinic for further examination who have a clear history of epidemiology, contact with suspected or confirmed patients, symptoms such as fever, cough, expectoration, and other symptoms. Otherwise, DF patients enter the routine specialist diagnosis and treatment process[8].DFU patients with fever or cough conduct 2019-nCoV nucleic acid test or chest CT test, consider suspected or confirmed COVID-19 who are directly admitted to the isolation ward by the fever clinic. The DF management medical team can assist in evaluation and guidance through the network system. Multiple disciplinary teams (MDT)should be reasonable to arrange consultation and treatment as efficiently as possible[9].MDT can make rational use of the network, telephone, WeChat, and remote consultation software equipment and other forms. DF patients with a high-risk foot (callus, verrucous process, blister, high arch foot, flatfoot or valgus, etc.) and no or mild infection (Wagner Grade 1) are recommended to the change wound dressing at home by the network clinic to reduce cross-infection. Medical staff can regularly contact patients through Wechat, telephone, and other ways, and assess wound conditions by photos, videos to guide wound treatment[10]. If the wound does not heal after the control of the epidemic, patients will go to the outpatient clinic for wound treatment or hospitalization. During the COVID-19 pandemic, the patients with Wagner Grades 2 5 wounds[11], severe limb ischemia (ankle-brachial index $\leq 0.4$ ), or unstable vital signs will go to the endocrinology department after excluding COVID-19[12].

The COVID-19 epidemic requires medical staff to adopt three levels of protection, with more special protection requirements for DFU patients. Since medical personnel often need to face the wounds of DFU patients, they are at risk of being contaminated by the patients' secretions and tissues during diagnosis and treatment. The level of protection for hands and face should be improved. According to the 
protection grade of the diabetic foot during the epidemic of COVID-19, all DFU patients should be regarded as potential suspected patients by at least the secondary protection. Intermediate protection is recommended when receiving patients with confirmed or suspected COVID-19 diabetic foot, blood collection, and wound treatment for DFU patients (whether with or without COVID-19) or surgery for patients with a non-COVID-19 diabetic foot. When performing surgery, sputum aspiration, intubation, and other operations for DFU patients who are diagnosed or suspected of the COVID-19, advanced protection of diabetic foot (the same as the third-level protection in general) are recommended [13-14].

Follow the suggestions and guidelines of relevant experts, this paper puts forward reasonable recommendations for the diagnosis and treatment of diabetic foot ulcers, optimize the diagnosis and treatment process, and reduce cross-infection in the hospital(Fig.1). The following three cases studies demonstrate its use in treatments and interventions. These patients underwent epidemiological history inquiries, temperature, blood routine examination, nucleic acid screening, and chest CT examination at the outpatient clinic. These patients were sent to our department after the exclusion of the COVID-19. Clinical photographs of the wounds before and after treatment were presented. Three patients with ulcerations in the hospital were discharged to home with outpatient care.

\section{Case Study 1}

A 61-year-old woman was admitted to our department with infected wounds that had been progressing for one month. In December 2019, the patient injured the first toe of her left foot due to careless manicure. The advent of the COVID-19 pandemic had resulted in the closing of some outpatient clinics for face-toface consultations. The patient applied the ointment by herself. One month later, the wound gradually developed swelling, fluid flow, stink and lose nails around the first toe of the left foot due to infection. In January 2020, the patient went to the local hospital for treatment, but her condition did not improve. Finally, on January 19,2020, the patient was admitted to our department for diagnosis and treatment. The patient's medical history included two years of diabetes poorly controlled with oral hypoglycemia medicine. Besides, the patient denied any previous disease history.

It was a penetrating wound that reached into the bone and corns of plantar. The wound area was about 3 $\mathrm{cm} \times 2 \mathrm{~cm}$ and involved first, second, and third toe of the left foot and one-thirds of the plantar area, covered with purulent discharge. Physical examination revealed redness, swelling, rupture, and exudation of the area surrounding the infected wound. (fig.2a,b). Bilateral dorsal pedis artery pulsation was symmetrical and normal.

After admission, the patient received antiinfection treatment and nibble debridement therapy. The patient and the wound were reassessed three days later. Considering the infection of wound and exudation of secretion after several debridements, VSD was used to treat the wound. The wound was explored after local anesthesia. After incision and drainage of the soft tissue abscess of the left foot, the necrotic tissue and pus in the sinus were thoroughly removed(Fig. 2c). Debridement and hemostasis were conducted to wash the wounds, and the whole wound covered by the VSD sponge was sealed with a thin film with 
biological permeability. The drainage area was completely isolated from the outside. Continuous negative pressure drainage was continued(Fig. 2d). After two weeks of VSD treatment, the patient's wound condition was significantly improved. The patient received debridement, dressing change treatment, and custom-made therapeutic footwear were used for assistance at the community ambulatory care center. Podiatrists follow up through the network platform, guide the change of dressing, and assist in the evaluation of wound changes. Ultimately, after 60 days of treatment, the patient's wound healed, and the sinus closed(Fig. 2e,f). Amputation was successfully avoided, and our therapy greatly improved the patient's quality of life.

\section{Case Study 2}

This case study describes a 47-year-old woman with a right leg ulcer. She had a medical history of diabetes(which was poorly controlled with insulin)and known peripheral neuropathy. She was admitted to hospital on April 10, 2020, with an ulcer of two month's duration. The ulcer, which was located on the lower $1 / 3$ of her right limb, measured $6 \times 4 \times 2 \mathrm{~cm}$ (length width depth) (Fig.3a). The presence of paraesthesia confirmed the diagnosis of peripheral neuropathy. Avascular assessment incorporating palpation of the dorsal pedis and lower limb vascular of color Doppler showed normal values. In February 2020 , the patient fell at home and injured the right calf. She had attempted to see her physician but was advised to stay home due to the COVID-19 pandemic, and was placed on oral antibiotics and guided the change of dressing by the physician. Subsequently, an abscess formed gradually within a month, with pain and swelling in the right leg. Upon evaluation, the wound had dark scabs, redness, swelling, significant tenderness, purulent drainage, and malodor. Foot arteriography confirmed the presence of right tibial osteomyelitis with soft tissue infection and rupture of calf accompanied by multiple small vascular (Fig. 3b).

She has started an intravenous infusion of moxifloxacin based on a drug-sensitivity test for two weeks. Abscess incision and nibbled debridement therapy were performed on the lower $1 / 3$ of her right limb to thoroughly remove necrotic tissue and pus, unhealthy tissue and skin were removed surrounding the infected wound. These treatments allowed to effectively evaluate clearing progress, prevented bleeding and helped induce the growth of fresh tissues in the wound. After thoroughly hemostasis, the wound was covered with a bio-film after placing a VSD sponge, and the negative pressure was applied at $0.02 \mathrm{kpa}$ (Fig. $3 \mathrm{C}$ ). The therapeutic schemes were modified based on the improvement of the wound and the skin around the wound. Over the next two weeks, The exudate volume had decreased, the healing trajectory progressed(Fig. 3d), as had the pain level, with the score 2/10, which had improved the patient's quality of life. The wound had decreased in size, and the calf lesion was on healing ultimately within eight weeks(Fig. 3e).

\section{Case Study 3}

A 71-year-old male with a history of type 2 diabetes, known peripheral neuropathy, arterial occlusion of the lower extremities, and artificial right femoral head arthroplasty attended to the hospital on May 10, 
2020 , with complaining of an increasingly painful in right toe over 10 days. The wound was producing viscous exudate and resulting in the maceration of the wound and peri-wound skin. The wound involved a grossly swollen right fifth toe and one-thirds of the dorsum of foot area, which was revealed scabs, swelling, redness, rupture, exudation, and covered with yellow-green purulent secretions(Fig. 4a). Physical examination included pulse palpation, which found a weak pulse on the right foot. Doppler ultrasound signals suggested some distal vascular disease.CT angiography (CTA) in the lower extremity artery confirmed the presence of occlusion of the right posterior tibial artery, severe stenosis of the right peroneal artery, and anterior tibial artery(Fig.4b,c).

A clinical diagnosis of diabetic foot ulcers with arterial sclerosis occlusion was made. Considering serious foot infection and toe necrosis and artery occlusion of the lower extremities, a partial amputation of the right fifth toe was performed in the clinic using local anesthesia(Fig. 4d). Simultaneously, this patient adopted antibiotic treatment, open therapy combined with dressings, and the use of negative pressure wound therapy. The infection was resolved within eight days, and his amputation sites began to heal. The patient presented with partial granulation tissue on the wound bed and healthy peri-wound skin. The patient had severe right peroneal and tibial artery stenosis, colleagues from the Vascular Surgery Department conducted artery balloon dilatation angioplasty of the right lower limb. During the pandemic, he was treated at home after discharge,followed up through the network platform and dressing changes by his attending physician(Fig. 4e). After one-and-a-half months, the wound was on a healing ultimately(Fig. 4f).

\section{Discussion}

At the time of the COVID-19 pandemic, priorities for inpatient care should focus on those with serious cases of the infection, such as diabetic foot ulcers. Of all the late stages of diabetes mellitus, those involving the foot have required more visits to the hospital to treat wounds by antibiotic therapy, debridement, and many other treatment modalities. The worrying nature of the above cases remind us of the possibility that there is the potential for a series of problems, resulting in major surgery, amputation, and mortality, to occur in the COVID-19 pandemic.DF patients to the stage of foot ulcers are often accompanied by a serious decline in systemic functions. The result may lead to susceptibility to 2019$\mathrm{nCoV}$ at the initial stage. The reaction may not be as severe as that of normal people in the middle and later stages. It will soon enter a critical state once it breaks out. In addition, DF is a late complication of diabetes mellitus often associated with diabetic peripheral neuropathy, which leads to the dullness of sensation and is easy to cover up and delay the disease. In order to improve the rescue rate of DF during the epidemic prevention and control period, enhance the self-protection awareness and ability of medical workers, and avoid cross-infection in hospitals, the first physician should strictly screen all patients with DF for COVID-19[15].

In recent years, as the aging of the population is increasing, the rates of DF rates and related amputations of DFU rates have also been increasing. Diabetic foot refers to a diabetic foot infection, ulcer, deep tissue destruction, distal peripheral vascular disease of the lower extremity, and local nerve abnormality, which 
will involve skin to bone and joint tissues. Its basic pathological mechanism is an infection, ischemia, and neuropathy, which cause gangrene, necrosis, and ulcer[16]. According to the etiology, the diabetic foot can be divided into neuroischemic diabetic foot and neurogenic diabetic foot. The main characteristics of neuroischemic diabetic foot are cool foot, no or weak pulsation of the dorsalis pedis artery, and less perfusion of peripheral circulation.

In the past, the treatment of DFU was based on the control of blood glucose, the thorough debridement of necrotic tissue, regular dressing change, and the use of effective antibiotics until the healing. Conventional repair methods were mainly based on the control of patients' blood glucose, which was treated with multiple debridements and dressing changes to control wound infection to promote wound healing. However, the treatment cycle was long, wound healing was slow, and the long-term use of antibiotics was prone to drug resistance, reinfection and rupture were easy to occur. For diabetic foot patients with Wagner grade 2 or above[17], it is often difficult to achieve the ideal therapeutic effect by simple medical or surgical treatment. Surgical debridement, wound closure, internal control of blood glucose, improvement of nerve function, and microcirculation are needed to achieve a better therapeutic effect. The latest introduction of negative pressure drainage technology has become a new treatment for diabetic foot patients[18].

In 1993, Dr. Fleischmann et al.[19] first reported that VSD technology was applied to the treatment of wound infection, and then gradually popularized in clinical application.VSD technology first uses medical foam and other unique materials to entirely cover or fill the skin and soft tissue defect wounds. After using the biological semipermeable membrane, the whole wound and the external environment are entirely isolated and sealed, and then a negative pressure drainage method is adopted. The technology can effectively remove wound necrosis tissue and exudate, and reduce the absorption of bacteria and toxins. Negative pressure drainage can compress the wound tissue to some extent, improve the local blood circulation of the wound, reduce the edema of the wound tissue, increase the blood supply of the wound tissue, promote the proliferation of capillaries and the growth of epithelial tissue, accelerate the formation of local granulation tissue of the wound, contribute to the reduction of the wound and promote the healing of the ulcer, and reduce the risk of amputation[20].

During VSD treatment, the wound and surrounding skin should be thoroughly disinfected and cleaned. According to the size and shape of the wound, VSD materials should be used to fill and cover the wound and cavity. During the filling process, there should be no space left, and the drainage tube should be directly led out from the wound. It should be noted that the coverage area of the semipermeable membrane at least $2 \mathrm{~cm}$ beyond the wound edge when using the biological semipermeable membrane to seal the wound surface, which is the critical step to maintain the success of negative pressure[21]. The negative pressure value should be maintained between $125 \sim 300 \mathrm{mmHg}(1 \mathrm{mmHg}=0.133 \mathrm{kpa})$, and conduct continuous negative pressure suction for 24 hours. The drainage tube is flushed with heparin saline every day to ensure smooth flow, and the condition of effective negative pressure suction and drainage fluid are observed. VSD dressings are changed every 4-6 days[22]. Once there is a visible secretion accumulation under the semipermeable membrane or VSD material recovers, it means that the 
negative pressure of VSD material disappears, and it should be replaced. One week is a course of treatment until fresh granulation tissue filling wound is ideal.

According to Wagner's classification, the above patients were classified as a grade 3 diabetic foot due to the presence of a deep abscess with colonized bacterial infection[17]. Apart from diabetes, no other underlying diseases such as hypertension and multiple organ failure were found in the patient, which was beneficial to wound healing. However, the blood supply aggravated the spread of infection. The success of this patient's treatment depended on antibiotic therapy combined with multiple debridements and dressing changes, and the concurrent use of dynamic complementary VSD methods. Antibiotics were used to prevent and control systemic infection in patients with wound infection and reduce the discomfort caused by severe reactions. Patients with diabetic foot complicated with mild and moderate infections are treated with antibiotics for 1-2 weeks, which is also recommended by the International Working Group on the Diabetic Foot (IWGDF) guidelines. Antibiotics should be paid attention to the selection and timing in the course of a patient's treatment, and avoid overuse to reduce the production of drug-resistant bacteria.

After admission, the wound with debridement and expansion was called occlusal debridement. The wound was gradually removed the necrotic tissue in several times, and add silver ion dressing between each debridement to promote the autolysis of necrotic material. In this way, it can help to obtain a relatively stable wound and avoid excessive damage to healthy tissues, and then fully retain the repairability of the wound itself.

Hydrogel and calcium alginate polymer as an inner dressing were used by the method of wet dressing change. Water gel, calcium alginate polymer, and epidermal growth factor were sprayed on the wound surface. Alginate has excellent hydrophilicity, which can be used together with hydrogel and epidermal growth factors to create a moist wound environment[23]. The wet liquid dressing is better than the traditional dressing, which can fully isolate bacteria and microorganisms in the external environment. At the same time, the moist wound environment is conducive to the absorption of bioactive substances in the dressing, to accelerate the autolysis of necrotic tissue, promote the growth of granulation tissue, and then accelerate the repair of the wound.

Mepilex is a kind of dressing with high water absorption, protection, and insulation[24].In our case, it was used as the outer dressing, which combined with the dressing selected by the inner layer. It can better maintain the moist environment of the wound and absorb the excessive secretion of the wound due to infection. Foam dressing is also more suitable for human body surface characteristics than general dressing. It can play a specific role in reducing pressure and prevent possible ischemia in the affected area. Besides, foam dressings do not need frequent replacement, generally can reach 1-week, and then enhance the patient's compliance.

After a period of in-hospital treatment, the wound of the patient improved. The patient carried out followup treatment and used therapeutic footwear. The application of therapeutic footwear ensures the leg movement of the patient, which is conducive to the regular blood supply of the wound. At the same time, 
it has a demonstrated plantar pressure-relieving effect during walking, prevents more severe lesions caused by the pressure of the wound, which is also recommended by the IWGDF guidelines[25].

\section{Conclusions}

During the epidemic period, how to make safe and effective protection, reduce the spread of hospitalrelated COVID-19, and optimize the diagnosis and treatment of diabetic foot ulcers is very important. Patients with diabetic foot ulcers should be achieved individual diagnosis and treatment by multidisciplinary cooperation, which can make patients get timely and reasonable treatment on the premise of prevention and control. The wound surface of diabetic foot ulcer with infection is usually large and complicated and often associated with sinus. The VSD method can effectively control the spread of wound infection of the diabetic foot, shorten the closing time of wound and sinus, improve the prognosis and reduce the risk of amputation, which is a safe and effective treatment.

\section{Declarations}

Ethics approval and consent to participate

This research was approved by the Medical Research Ethics Committee of Second Affiliated Hospital of Fujian Medical University, Quanzhou, P. R. China. All participants gave written consent after being informed about the procedure.

Consent for publication

All authors have reviewed the final version of the manuscript and agree with the publication of the information presented here.

Availability of data and materials

The datasets used during the current study are available from the corresponding author on reasonable request.

Competing interests

No potential conflicts of interest relevant to this article were reported.

Funding

This study was supported by the startup fund for scientific research, Fujian Medical University (2020QH1122) to JYZ, the Startup Fund for scientific research, Fujian Medical University (2018QH1105) to JYL, Fujian Province Health Science and Technology Project (2021TG014) to JYL.

Authors' contributions

Jiayu Lin conceived this project and supervised the experiments. Jinying Zhang analyzed the data and wrote the paper.

Acknowledgements 
This study was supported from the Second Affiliated Hospital of Fujian Medical University. We thank all of the participants in the study for their cooperation in this study.

\section{References}

[1] Schaper NC,Van Netten JJ,Apelqvist J,et al.Prevention and management of foot problems in diabetes:a Summary Guidance for Daily Practice 2015,based on the IWGDF Guidance Documents[J].Diabetes Metab Res Rev,2016,32(Suppl 1):7-15.

[2] Holman N,Young B,Stephens $\mathrm{H}$,et al.Pilot study to assess measures to be used in the prospective audit of the management of foot ulcers in people with diabetes[J].Diabetic Medicine,2015,32(1):78-84.

[3]Margolis DJ, Malay DS, Hoffstad OJ, et al. Incidence of diabetic foot ulcer and lower extremity amputation among medicare beneficiaries, 2006 to 2008[M]. Rockville (MD):Agency for Healthcare Research and Quality (US), 2011.

[4]Wukich DK, Raspovic KM, Suder NC. Patients with diabetic foot disease fear major lower-extremity amputation more than death[J]. Foot Ankle Spec, 2018, 11(1): 17-21.

[5]Lipsky, B. A., et al. (2012). "2012 Infectious Diseases Society of America clinical practice guideline for the diagnosis and treatment of diabetic foot infections." Clin Infect Dis 54(12): e132-173.

[6]Li, Z., et al. (2017). "Effect of vacuum sealing drainage in dermatoplasty of large area of cutaneous defects." Int J Surg 42:143-146.

[7] COVID-19 Epidemiology Group of Emergency response Mechanism, Chinese Center for Disease Control and Prevention. Analysis of epidemiological characteristics of COVID-19 [J]. Chinese Journal of Epidemiology, 2020, 41 (2): 145-151.

[8]General Office of the State Health Commission and Office of the State Administration of traditional Chinese Medicine. Notice on issuing COVID-19 's diagnosis and treatment plan (trial version 7)[Z].202003-04.

[9]Huizing E, Schreve MA, Kortmann W, et al. The effect of a multi-disciplinary outpatient team approach on outcomes in diabetic foot care: a single center study. J Cardiovasc Surg(Torino), 2019, 60(6): 662-671.

[10]General Office of the State Health Commission. Notice on the issuance of guidelines for the prevention and control of infection in home isolation medical observation in the prevention and control of pneumonia infected by novel coronavirus[Z].2020-02-23

[11]Diabetes Branch of Chinese Medical Association, Infectious Diseases Branch of Chinese Medical Association, tissue repair and Regeneration Branch of Chinese Medical Association. Chinese guidelines for prevention and treatment of Diabetic foot (2019 Edition) ( $($ ) [J]. Chinese Journal of Diabetes, 2019,11 (4): 238-247. 
[12]Diabetes Branch of Chinese Medical Association, Infectious Diseases Branch of Chinese Medical Association, tissue repair and Regeneration Branch of Chinese Medical Association. Chinese guidelines for prevention and treatment of Diabetic foot (2019 Edition) (『) [J]. Chinese Journal of Diabetes, 2019. 11 (3): 161-189.

[13]Organization WH. Infection prevention and control during health care when novel coronavirus (nCoV) infection is suspected: interim guidance, 2020 January 25.

[14]National Health Office Medical letter (2020) 75, guidelines for the use of common medical protective equipment in the prevention and control of pneumonia infected by novel coronavirus (trial). Beijing: general Office of the State Health Commission, 2020.

[15]Diabetic Branch of Chinese Medical Association. Suggestions on the management of diabetic patients complicated with COVID-19 [J]. Chinese Journal of Diabetes, 20200.12 (2): 73-75.

[16]Singh N,Armstrong DG,Lipsky BA.Preventing foot ul[1]cers in patients with diabetes[J]. Jama,2005,293(2):217-228.

[17]Wagner FWJr. The dysvascular foot: a system of diagnosis and treatment. Foot Ankle 1981;2:64122.

[18]Huang, Q., et al. (2019). "Comparison of Vacuum Sealing Drainage and Traditional Therapy for Treatment of Diabetic Foot Ulcers: A Meta-Analysis." J Foot Ankle Surg 58(5): 954-958.

[19]Fleischmann W,Strecker W,Bombelli M.Vacuum sealing as treatment of soft damage in open fractures[J].Der Unfallchirurg,1993,96(7):488-492.

[20]Hui Zhou, Xiaoping Yang, Yulei Gao, et al. Treatment of diabetic foot ulcer by vacuum sealing drainage [J]. Chinese Journal of Orthopaedic Surgery, 2019,27 (13): 1244-1245.

[21]Jian Song, Aixi Yu, Baiwen Qi, et al. application of vacuum sealing drainage technology in the treatment of refractory ulcer wounds [J]. Chinese Journal of microsurgery, 2016, 39 (1): 102-104.

[22]Liu, Z., et al. (2018). "Negative pressure wound therapy for treating foot wounds in people with diabetes mellitus." Cochrane Database Syst Rev 10: CD010318..

[23]Varaprasad, K., et al. (2020). "Alginate-based composite materials for wound dressing application:A mini review." Carbohydr Polym 236: 116025.

[24]Weller, C. D., et al. (2020). "First-Line Interactive Wound Dressing Update: A Comprehensive Review of the Evidence." Front Pharmacol 11: 155.

[25]Bus, S. A., et al. (2020). "Guidelines on the prevention of foot ulcers in persons with diabetes (IWGDF 2019 update)." Diabetes Metab Res Rev 36 Suppl 1: e3269. 


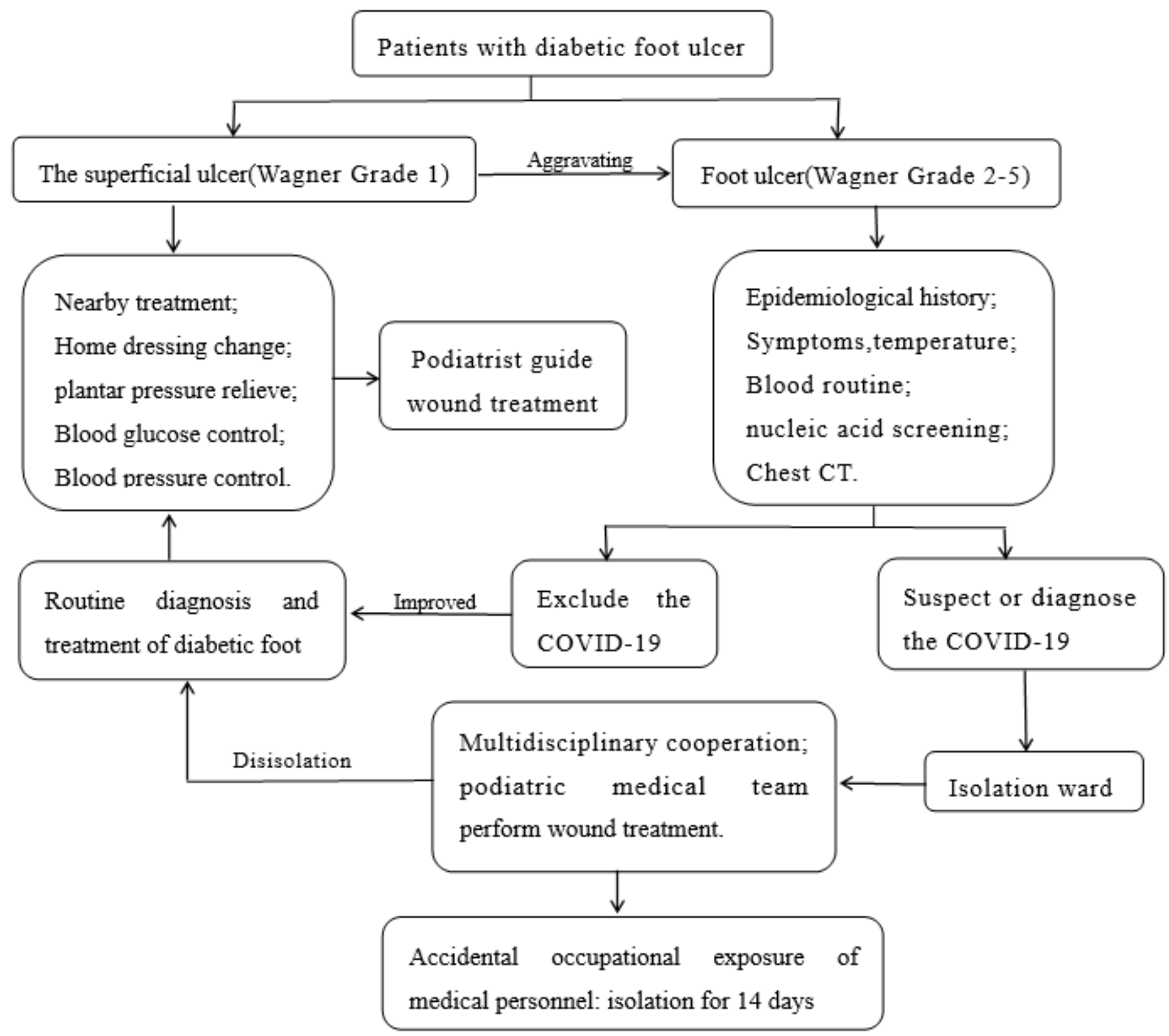

Figure 1

Flow chart of patients with diabetic foot ulcer treatment during COVID-19 epidemic 

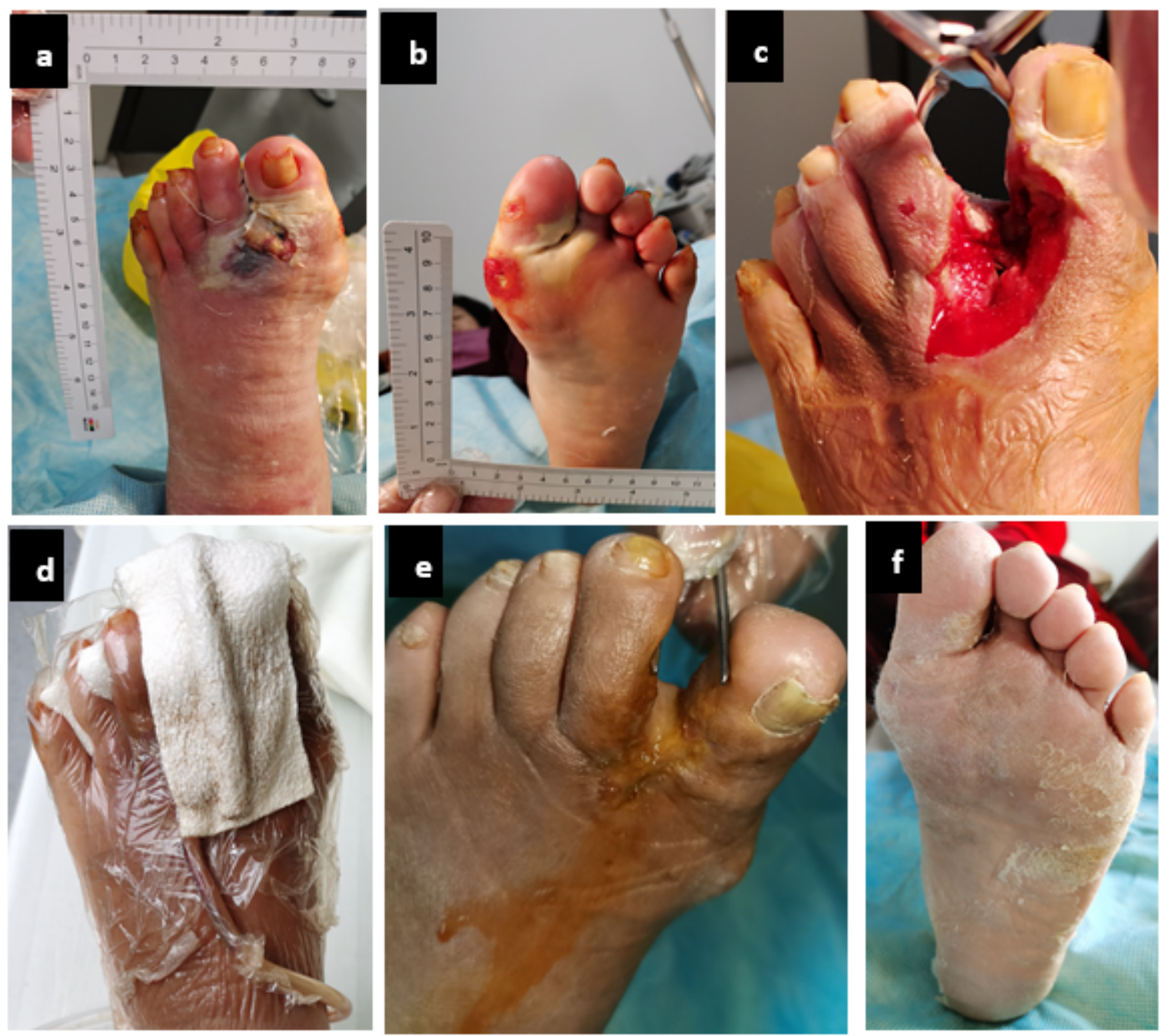

Figure 2

Case study 1. Diabetic foot (DF) condition after admission (a,b); the wound after antinfection treatment and occlusal debridement therapy (c); vacuum sealing drainage (d); wound closure at 60 days (e,f) 

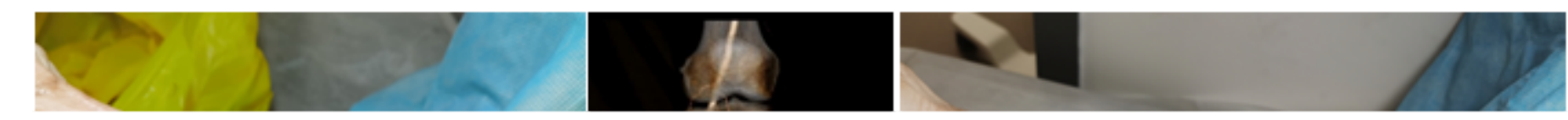

\section{Figure 3}

Case study 2. Diabetic foot (DF) condition after admission (a);vascular images and showing osteomyelitis of the lower right limb (b); vacuum sealing drainage and after debridement (c,d); wound closure at 2 months (e)

\section{Figure 4}

Case study 3. Diabetic foot (DF) condition after admission (a);vascular images and showing occlusion of right posterior tibial artery,severe stenosis of right peroneal artery and anterior tibial artery $(b, c)$; after debridement and vacuum sealing drainage $(d, e)$; wound closure at one-and-a-half months ( $f$ ) 